\title{
A Bayesian approach for the evaluation of six diagnostic assays and the estimation of Cryptosporidium prevalence in dairy calves
}

\author{
Thomas GEURDEN*, Dirk BERKVENS, Peter GELDHOF, \\ Jozef VERCRUYSSE, Edwin ClAEREBOUT
}

Laboratory of Parasitology, Faculty of Veterinary Medicine, Ghent University, Salisburylaan 133, 9820 Merelbeke, Belgium

(Received 9 December 2005; accepted 6 March 2006 )

\begin{abstract}
The prevalence of Cryptosporidium in calves and the test properties of six diagnostic assays (microscopy (ME), an immunofluorescence assay (IFA), two ELISA and two PCR assays) were estimated using Bayesian analysis. In a first Bayesian approach, the test results of the four conventional techniques were used: ME, IFA and two ELISA. This four-test approach estimated that the calf prevalence was $17 \%$ (95\% Probability Interval (PI): 0.1-0.28) and that the specificity estimates of the IFA and ELISA were high compared to ME. A six-test Bayesian model was developed using the test results of the 4 conventional assays and 2 PCR assays, resulting in a higher calf prevalence estimate (58\% with a 95\% PI: $0.5-0.66$ ) and in a different test evaluation: the sensitivity estimates of the conventional techniques decreased in the six-test approach, due to the inclusion of two PCR assays with a higher sensitivity compared to the conventional techniques. The specificity estimates of these conventional assays were comparable in the four-test and six-test approach. These results both illustrate the potential and the pitfalls of a Bayesian analysis in estimating prevalence and test characteristics, since posterior estimates are variables depending both on the data at hand and prior information included in the analysis. The need for sensitive diagnostic assays in epidemiological studies is demonstrated, especially for the identification of subclinically infected animals since the PCR assays identify these animals with reduced oocyst excretion, which the conventional techniques fail to identify.
\end{abstract}

Cryptosporidium / Bayesian / test evaluation / prevalence / calves

\section{INTRODUCTION}

Cryptosporidium is a protozoan parasite closely related to the gregarine parasites within the Apicomplexa [3] and is considered as an important agent in the aetiology of diarrhoea in both humans and livestock $[7,15]$. In cattle, the parasite has been associated both with acute and chronic

* Corresponding author: thomas.geurden@ugent.be clinical disease [23], especially in young calves [4]. The prevalence of $C$. parvum in calves aged less than 6 months varies from $2.4 \%$ [38] to $54 \%$ [27]. This variation in prevalence is not only due to substantial differences in climatological or management conditions, but also to differences in study design, such as differences in the number of farms or animals, the age of the animals under study, sampling strategies or the diagnostic techniques used in the study. The conventional diagnostic assays for the 
diagnosis of Cryptosporidium in calves comprise microscopic examination (ME) following Kinyoun acid fast staining, modified Ziehl-Neelsen staining or carbolfuchsine staining, and immunological assays, such as the immunofluorescence assay (IFA) and enzyme-linked immunosorbent assay (ELISA). More recently, polymerase chain reaction (PCR) has been shown to be both very sensitive and specific [19]. Accuracy assessment of diagnostic tests is traditionally performed using contingency tables with the test results of one assay or a combination of assays [14] as the gold standard reference. However, due to a lack of knowledge of the true disease status of the tested animals (i.e. no gold standard), diagnostic test evaluation is frequently rendered ineffective leading to an over-or underestimation of the performance of the new test. The accuracy assessment of tests for the diagnosis of Cryptosporidium is illustrative of the problem. In some studies ME was used as the gold standard, overrating the ME sensitivity and specificity values compared to ELISA or IFA [13, 21], whereas in other studies IFA [27] or PCR [19] were used as the gold standard, underestimating the relative sensitivity and specificity of ME. Similar reflections can be made for the evaluation of ELISA using IFA as the gold standard $[8,30]$. These problems can be partially evaded by using spiked samples to evaluate the sensitivity and specificity of diagnostic test methods. A higher recovery rate for IFA compared to ME has been described [39, 40], although recovery of Cryptosporidium oocysts from spiked specimens is known to be low [39]. Furthermore, this procedure can only be used in a controlled evaluation protocol and not for evaluation of diagnostic tests in clinical or epidemiological studies where the true disease status of the subject is unknown. Recently, Bayesian analysis has proven its value in circumventing this gold standard problem $[1,5,11,26]$. In this study, two different Bayesian approaches are presented for the estimation of Cryptosporidium prevalence in dairy calves in the province of East-Flanders, Belgium, and for the evaluation of six diagnostic tests.

\section{MATERIALS AND METHODS}

\subsection{Study design}

Fifty dairy farms in the province of East-Flanders, Belgium, were randomly selected and visited on a single occasion between September 2001 and November 2002. All calves were Holstein or Holstein cross. Faecal specimens were collected rectally from all calves aged from newborn to 10 weeks, present on the farm at the time of the visit. All samples were transported to the laboratory and examined using six different diagnostic assays. The samples were examined within $48 \mathrm{~h}$ after collection with ME and IFA. Afterwards, all samples were preserved at $-20{ }^{\circ} \mathrm{C}$ and examined with ELISA and PCR.

\subsection{Detection of Cryptosporidium oocysts}

\subsubsection{The carbolfuchsine smear method followed by microscopical examination}

A direct faecal smear followed by carbolfuchsine staining [12] was used to demonstrate Cryptosporidium oocysts in fresh faecal material. Approximately $2 \mathrm{~cm}^{2}$ of each slide was examined at a $1000 \times$ magnification. A sample was considered positive as soon as an oocyst with the correct morphology $(4.6-5.6 \mu \mathrm{m} \times 4-4.8 \mu \mathrm{m})$ was identified.

\subsubsection{Immunofluorescence Assay}

The quantitative IFA was developed using the commercial MERIFLUOR Cryptosporidium kit (Meridian Diagnostics, 
Inc., Cincinnati, Ohio, USA) and based on the method of Xiao and Herd [41]: one gram of faeces was suspended in distilled water and strained through a layer of surgical gauze to withold large debris. After sedimentation for at least $1 \mathrm{~h}$ and centrifugation at $3000 \mathrm{~g}$ for $5 \mathrm{~min}$, the sediment was resuspended in $1 \mathrm{~mL}$ of distilled water. After thorough vortexing, an aliquot of $10 \mu \mathrm{L}$ was pipetted onto a treated IFAslide. After staining the slide, as instructed by the manufacturer, the entire slide was examined at a $400 \times$ magnification under a fluorescence microscope. A sample was considered positive if at least one, clearly recognisable Cryptosporidium oocyst was identified. The number of Cryptosporidium oocysts per gram faeces was obtained by multiplying the total number of oocysts on the slide by 100 .

\subsubsection{Enzyme-linked immunosorbent assay for the detection of Cryptosporidium antigen in the faeces}

The TechLab Cryptosporidium test (Techlab, Inc., Blacksburg, VA, USA) and the Tetrakit or Bio-X Digestive ELISA Kit (Bio-X diagnostics, Marche-en-Famenne, Belgium) were used to demonstrate the prevalence of Cryptosporidium antigen in frozen samples. Both assays were used as instructed by the manufacturer.

\subsubsection{PCR diagnostic assays (PCR)}

DNA was extracted from faecal samples using the QIAamp ${ }^{\circledR}$ Stool Mini Kit (Qiagen, Hilden, Germany). The eluted DNA was subsequently precipitated by the addition of $140 \mu \mathrm{L}$ isopropanol and centrifuged for $30 \mathrm{~min}$ at $16000 \mathrm{~g}$. Following a wash step with $75 \%$ ethanol, the pellet was dissolved in $12.5 \mu \mathrm{L}$ ultrapure water. Amplification products of both PCR assays were randomly selected and sequenced throughout the study to confirm the specificity of both PCR assays.

\subsubsection{1. $C-P C R$}

The oligonucleotide primers (CP-CR and $\mathrm{O} 21 \mathrm{~F}$ ) used for detection of Cryptosporidium DNA were previously described $[17,18]$. They were designed to amplify a 312 bp product from the bovine $C$. parvum genotype. The reaction mixture consisted of a master mix containing Taq DNA polymerase, dNTP mixture, Bovine Serum Albumin, standard Taq buffer, $10 \mathrm{pM}$ of each primer and $2.5 \mu \mathrm{L}$ of template cDNA in a total of $25 \mu \mathrm{L}$. Subsequent steps were initial denaturation for 2 min at $94{ }^{\circ} \mathrm{C}$, followed by 40 cycles of denaturation for $30 \mathrm{~s}$ at $94{ }^{\circ} \mathrm{C}$, annealing for $30 \mathrm{~s}$ at $58{ }^{\circ} \mathrm{C}$ and an extension for $30 \mathrm{~s}$ at $72{ }^{\circ} \mathrm{C}$. Amplification products were subsequently visualised on $1.5 \%$ agarose gels with ethidium bromide. A sample was considered positive if a band of $312 \mathrm{bp}$ was visualised. A positive and negative control sample was included in each PCR reaction. The positive control consisted of a plasmid containing the $312 \mathrm{bp}$ rDNA product. This was originally cloned from a $C$. parvum DNA sample isolated from a $C$. parvum positive calf (kindly provided by Simone Caccio). The negative control was purified water.

\subsubsection{COWP-PCR}

The oligonucleotide primers (cry-15 and cry-9) used for detection of the COWP were previously described by [33]. They were designed to amplify a 550 bp product from the $C$. parvum genome. The reaction mixture is similar to the previous protocol. Subsequent steps were 40 cycles of denaturation for $50 \mathrm{~s}$ at $94{ }^{\circ} \mathrm{C}$, annealing for $30 \mathrm{~s}$ at $55^{\circ} \mathrm{C}$ and an extension for $50 \mathrm{~s}$ at $72^{\circ} \mathrm{C}$. 


\subsection{Statistical analysis}

The apparent prevalence for each diagnostic assay was calculated as the number of positive animals divided by the total number of animals. A Bayesian analysis framework was used to draw inferences about the prevalence of Cryptosporidium and the test properties (sensitivity and specificity) of the different tests. Different models were constructed in WinBUGS 1.4 [35]. Typically, a burn-in phase of 5000 iterations was used and the models were run for another 10000 iterations to obtain estimates. The Brooks, Gelman and Rubin convergence statistic [9] was used to assess model convergence and only properly converged models were further considered. Model selection proceeded on grounds of identifiability of the model and through minimisation of the deviance information criterion (DIC) while ensuring a positive number of parameters $\left(p_{D}\right)$ estimated in the model $[10,34]$. Furthermore, the Bayesian $p$-value [10] was used as a measure for the goodness-of-fit of the model (a comparison of the data to the posterior predictive distribution) and essentially represents the proportion of positive differences at consecutive iterations between the deviance for "cell" counts, sampled from the model, and the deviance calculated for the data (a value close to 0.50 signifies a model that does not exhibit lack-of-fit). Since the independence model does not take possible conditional test characteristics into account [11], only fully conditional models were considered. Two different approaches were used to estimate the prevalence and test characteristics. In the four-test approach, a model, based on a multinomial distribution and including all possible interactions between the ME, IFA, Techlab and Tetra was used, requiring 31 parameters to be estimated [5]. Furthermore, a new six-test approach was developed, based on the results of all six individual tests, requiring 127 parameters to be estimated: the prevalence, the sensitivity and specificity of the first test, two conditional sensitivities and two conditional specificities for the second test, four conditional sensitivities and four conditional specificities for the third test, eight conditional sensitivities and eight conditional specificities for the fourth test, sixteen conditional sensitivities and sixteen conditional specificities for the fifth test and finally thirty-two conditional sensitivities and thirty-two conditional specificities for the sixth test (cf. conditional probabilities, P. 675). This model is in fact not identifiable, since the data only allow 63 parameters to be estimated (64 "classes" of test results with probabilities summing to unity). Therefore, the model building strategy consisted of incorporating extraneous prior information in a dependence model, such as expert opinion [36], in an attempt to reduce the number of parameters to be estimated. This can be done by stating, for example, that the specificity of one of the tests is 1, leading to the exclusion of all parameters pertaining to false positives for this test: e.g. in Conditional probabilities, P. 675, equating the specificity of test 1 to $1(\mathrm{P} 3=1)$ automatically means that $\mathrm{P} 7$ and all parameters including $\mathrm{P} 7$ do not need to be estimated, since $\operatorname{Pr}\left(D^{-} \cap T_{1}^{+}\right)=0$. For other parameters, no objective prior information can be formulated. Therefore it is necessary to leave prior information on these parameters non-informative, if one wants to maintain a minimum degree of honesty [10]. All prior information that was used in the present study, is based on expert opinion or previously published data and is therefore empirical information. No data of the present study were used to formulate these priors.

Prior information can also be applied to reduce the possible range of values for a specific parameter [6]. This reduction may affect the possible range of values for other parameters as well. Because of the 


\section{CONDITIONAL PROBABILITIES}

\begin{tabular}{|c|c|}
\hline P1 Prevalence & $\operatorname{Pr}\left(D^{+}\right)$ \\
\hline $\mathrm{P} 2 \mathrm{Se}_{1}$ & $\operatorname{Pr}\left(T_{1}^{+} \mid D^{+}\right)$ \\
\hline $\mathrm{P} 3 \mathrm{Sp}_{1}$ & $\operatorname{Pr}\left(T_{1}^{-} \mid D^{-}\right)$ \\
\hline P4 & $\operatorname{Pr}\left(T_{2}^{+} \mid D^{+} \cap T_{1}^{+}\right)$ \\
\hline P5 & $\operatorname{Pr}\left(T_{2}^{+} \mid D^{+} \cap T_{1}^{-}\right)$ \\
\hline P6 & $\operatorname{Pr}\left(T_{2}^{-} \mid D^{-} \cap T_{1}^{-}\right)$ \\
\hline P7 & $\operatorname{Pr}\left(T_{2}^{-} \mid D^{-} \cap T_{1}^{+}\right)$ \\
\hline P8 & $\operatorname{Pr}\left(T_{3}^{+} \mid D^{+} \cap T_{1}^{+} \cap T_{2}^{+}\right)$ \\
\hline P9 & $\operatorname{Pr}\left(T_{3}^{+} \mid D^{+} \cap T_{1}^{+} \cap T_{2}^{-}\right)$ \\
\hline P10 & $\operatorname{Pr}\left(T_{3}^{+} \mid D^{+} \cap T_{1}^{-} \cap T_{2}^{+}\right)$ \\
\hline P11 & $\operatorname{Pr}\left(T_{3}^{+} \mid D^{+} \cap T_{1}^{-} \cap T_{2}^{-}\right)$ \\
\hline P12 & $\operatorname{Pr}\left(T_{3}^{-} \mid D^{-} \cap T_{1}^{-} \cap T_{2}^{-}\right)$ \\
\hline $\mathrm{P} 13$ & $\operatorname{Pr}\left(T_{3}^{-} \mid D^{-} \cap T_{1}^{-} \cap T_{2}^{+}\right)$ \\
\hline P14 & $\operatorname{Pr}\left(T_{3}^{-} \mid D^{-} \cap T_{1}^{+} \cap T_{2}^{-}\right)$ \\
\hline P15 & $\operatorname{Pr}\left(T_{3}^{-} \mid D^{-} \cap T_{1}^{+} \cap T_{2}^{+}\right)$ \\
\hline P16 & $\operatorname{Pr}\left(T_{4}^{+} \mid D^{+} \cap T_{1}^{+} \cap T_{2}^{+} \cap T_{3}^{+}\right)$ \\
\hline \multicolumn{2}{|l|}{$\ldots$} \\
\hline $\mathrm{P} 23$ & $\operatorname{Pr}\left(T_{4}^{+} \mid D^{+} \cap T_{1}^{-} \cap T_{2}^{-} \cap T_{3}^{-}\right)$ \\
\hline P24 & $\operatorname{Pr}\left(T_{4}^{-} \mid D^{-} \cap T_{1}^{-} \cap T_{2}^{-} \cap T_{3}^{-}\right)$ \\
\hline P25 & $\operatorname{Pr}\left(T_{4}^{-} \mid D^{-} \cap T_{1}^{-} \cap T_{2}^{-} \cap T_{3}^{+}\right)$ \\
\hline \multicolumn{2}{|l|}{$\ldots$} \\
\hline P31 & $\operatorname{Pr}\left(T_{4}^{-} \mid D^{-} \cap T_{1}^{+} \cap T_{2}^{+} \cap T_{3}^{+}\right)$ \\
\hline P32 & $\operatorname{Pr}\left(T_{5}^{+} \mid D^{+} \cap T_{1}^{+} \cap T_{2}^{+} \cap T_{3}^{+} \cap T_{4}^{+}\right)$ \\
\hline \multicolumn{2}{|l|}{$\ldots$} \\
\hline P50 & $\operatorname{Pr}\left(T_{5}^{-} \mid D^{-} \cap T_{1}^{-} \cap T_{2}^{-} \cap T_{3}^{+} \cap T_{4}^{-}\right)$ \\
\hline P51 & $\operatorname{Pr}\left(T_{5}^{-} \mid D^{-} \cap T_{1}^{-} \cap T_{2}^{-} \cap T_{3}^{+} \cap T_{4}^{+}\right)$ \\
\hline \multicolumn{2}{|l|}{$\ldots$} \\
\hline P63 & $\operatorname{Pr}\left(T_{5}^{-} \mid D^{-} \cap T_{1}^{+} \cap T_{2}^{+} \cap T_{3}^{+} \cap T_{4}^{+}\right)$ \\
\hline P64 & $\operatorname{Pr}\left(T_{6}^{+} \mid D^{+} \cap T_{1}^{+} \cap T_{2}^{+} \cap T_{3}^{+} \cap T_{4}^{+} \cap T_{5}^{+}\right)$ \\
\hline \multicolumn{2}{|l|}{$\ldots$} \\
\hline P103 & $\operatorname{Pr}\left(T_{6}^{-} \mid D^{-} \cap T_{1}^{-} \cap T_{2}^{-} \cap T_{3}^{+} \cap T_{4}^{+} \cap T_{5}^{+}\right)$ \\
\hline P104 & $\operatorname{Pr}\left(T_{6}^{-} \mid D^{-} \cap T_{1}^{-} \cap T_{2}^{+} \cap T_{3}^{-} \cap T_{4}^{-} \cap T_{5}^{-}\right)$ \\
\hline \multicolumn{2}{|l|}{$\ldots$} \\
\hline P127 & $\operatorname{Pr}\left(T_{6}^{-} \mid D^{-} \cap T_{1}^{+} \cap T_{2}^{+} \cap T_{3}^{+} \cap T_{4}^{+} \cap T_{5}^{+}\right)$ \\
\hline
\end{tabular}


Table I. The apparent prevalence and the test properties of the diagnostic tests (95\% PI) as estimated by the four test approach and the six test approach.

\begin{tabular}{lcccccc}
\hline & \multicolumn{3}{c}{4 test approach } & & \multicolumn{2}{c}{6 test approach } \\
\cline { 2 - 4 } \cline { 7 - 7 } & AP & Se $(\%)$ & Sp $(\%)$ & & Se $(\%)$ & Sp $(\%)$ \\
\hline C-PCR & 46 & ND & ND & & $79(69-87)$ & 100 \\
COWP PCR & 35 & ND & ND & & $59(50-67)$ & 100 \\
ME & 30 & $78(56-95)$ & $79(72-87)$ & & $40(31-49)$ & $84(75-97)$ \\
IFA & 16 & $78(54-95)$ & $95(91-99)$ & & $26(19-34)$ & $94(88-99)$ \\
Techlab & 21 & $76(54-92)$ & $89(84-94)$ & & $37(28-46)$ & $84(76-92)$ \\
Tetra & 14 & $59(39-77)$ & $93(89-96)$ & & $30(21-42)$ & $88(81-94)$ \\
\hline
\end{tabular}

$\mathrm{AP}=$ apparent prevalence; $\mathrm{Se}=$ sensitivity $\mathrm{Sp}=$ specificity $\mathrm{ND}=$ not done .

complexity of the model, it is impossible to predict or calculate what level of reduction in the number of parameters to be estimated will result from this prior information. Therefore, DIC is used to assist in model selection. DIC is an information criterion and consists of two components: first an equivalent of a likelihood measure (transformed in such a way that a lower value means a better fit) and secondly a penalty for the complexity of the model (the lower the value, the simpler the model). This DIC is to be minimised during the model building process, attempting to find the best fit with the simplest possible model. The number of parameters $\left(p_{D}\right)$ that was effectively estimated in the model represents the complexity of the model and is an indication of the final reduction in the number of parameters that had to be estimated.

\section{RESULTS}

\subsection{Animals}

In total, 234 calves were examined for the presence of Cryptosporidium oocysts in their faeces. The mean age was $26.2 \pm 1.4$ (mean \pm standard error) days ranging from 1 to 70 days. More than $60 \%$ of the calves were younger than 1 month. Seventy-three percent of the investigated animals were female and $27 \%$ were male. There were an average number of 5 calves on the farms, ranging from 1 to 14 .

\subsection{Apparent calf prevalence and Bayesian analysis}

The apparent calf prevalence as estimated by the different diagnostic assays, is presented in Table I and ranged from 0.14 in the Tetra ELISA up to 0.46 in the C-PCR. In the four-test approach, a conditionally dependent test model was used. Prior information on prevalence (prevalence constrained between 0.1-0.8) and on the specificity of the IFA, Techlab and Tetra (0.7-1) had to be included into the model to reach convergence. Adding information on the sensitivity of the IFA (0.4-1) further improved the model. Additional constraints did not, however, improve the model (Tab. II). Therefore, this model was used to estimate prevalence and test characteristics of the four assays. The estimated prevalence was 0.17 (95\% Probability Interval (PI): 0.1-0.28). The estimated sensitivity and specificity of each test are presented in Table I.

Since a six-test dependence model is in fact not identifiable due to a high number of parameters that have to be estimated compared to the degrees of freedom offered by the dataset, prior information on 
Table II. Prevalence (95\% PI) and model comparison in the 4 and the 6 test approach based on the deviance information criterion (DIC), the number of parameters $\left(p_{D}\right)$, and a Bayesian $P$-value (Bayes- $p$ ).

\begin{tabular}{lcccc}
\hline & Bayes- $p$ & $p_{D}$ & DIC & $P$ \\
\hline 4 test approach & \multicolumn{4}{c}{ Model did not converge } \\
Dependent $\mathrm{P}=0.1-0.8 ; \mathrm{Sp}_{2}=0.7-1$ & \multicolumn{4}{c}{ Model did not converge } \\
Dependent $\mathrm{P}=0.1-0.8 ; \mathrm{Sp}_{2,3}=0.7-1$ & 0.5451 & 8.582 & 71.481 & $0.198(0.11-0.47)$ \\
Dependent $\mathrm{P}=0.1-0.8 ; \mathrm{Sp}_{2,3,4}=0.7-1$ & 0.5642 & 9.582 & 70.939 & $0.167(0.11-0.26)$ \\
Dependent $\mathrm{P}=0.1-0.8 ; \mathrm{Sp}_{2,3,4}=0.7-1 ; \mathrm{Se}_{2}=0.4-1$ & & \\
Dependent $\mathrm{P}=0.1-0.8 ; \mathrm{Sp}_{2,3,4}=0.7-1 ; \mathrm{Se}_{2,3}=0.4-1$ & 0.7152 & 9.011 & 77.08 & $0.149(0.10-0.21)$ \\
Dependent $\mathrm{P}=0.1-0.8 ; \mathrm{Sp}_{2,3,4}=0.7-1 ; \mathrm{Se}_{2,3,4}=0.4-1$ & 0.5288 & 7.263 & 79.83 & $0.137(0.10-0.19)$ \\
\hline
\end{tabular}

6 test approach

Dependent

Model is not identifiable

Dependent $\mathrm{Sp}_{P C R 1 \text { and } 2}=1$

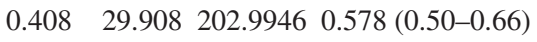

$\mathrm{P}=$ prevalence $\mathrm{Sp}=$ specificity $\mathrm{Se}=$ sensitivity $; 2=\mathrm{IFA} ; 3=$ Techlab ELISA; 4 = Tetrakit ELISA; PCR 1 = C-PCR; PCR 2 = COWP PCR.

the specificity of both PCR assays ( $\mathrm{sp}=1)$ was included $[18,33]$. The specificity of both PCR assays was confirmed by the sequencing of amplification products of randomly chosen samples throughout the study $(n=25)$. All sequences proved to be $C$. parvum, both for the C-PCR and the COWP-PCR. The specificity constraints made the model converge, whereas widening the constraints on the specificity of both PCR assays to a range between 0.8 and 1 prohibited the model convergence. Therefore the model with the more rigid constraints on PCR specificity ( $\mathrm{sp}=1$ ) was used to estimate the test characteristics of all diagnostic assays (Tab. I). The animal population prevalence estimated using this model was 0.58 (95\% PI: 0.5-0.66).

\subsection{Intensity of oocyst excretion and average prevalence in different age categories}

For all the tests, a high number of positive calves was found between the age of 1 and 4 weeks. In calves older than one month, the number of positive calves decreased, except for the PCR as- says, demonstrating a high number of positive test results in calves up to the age of 10 weeks. The apparent prevalence in calves younger and older than one month as estimated by the C-PCR was $45 \%$ and $49 \%$ respectively. For the COWP the prevalence was $34 \%$ and $41 \%$ respectively for calves younger and older than one month.

Since the number of positive calves for the different age categories estimated by ME, IFA and both ELISA were similar, only the data obtained with IFA are presented in Figure 1, in comparison with the data of the C-PCR assay. The number of excreted oocysts was determined on the same samples using IFA and ranged from 200 to 24000000 oocyst per gram faeces (OPG). The OPG was the highest in calves between the age of 1 to 3 weeks (Fig. 1). The highest individual OPG was observed in a 12 day old calf.

\section{DISCUSSION}

This is the first known study to use a Bayesian approach to estimate the 


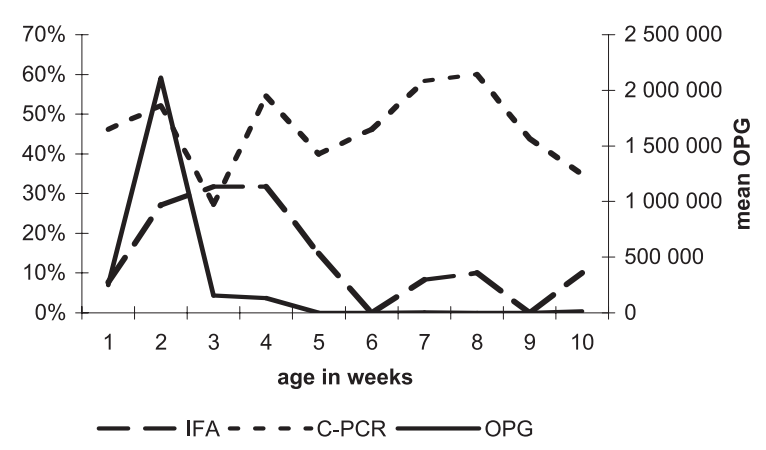

Figure 1. Prevalence and mean oocyst excretion (oocysts per gram faeces: OPG) of Cryptosporidium parvum in different age categories (1-10 weeks), as diagnosed by IFA and the C-PCR assay.

prevalence of Cryptosporidium in dairy calves and to evaluate diagnostic assays for the detection of the parasite. Since there is no gold standard for the diagnosis of a Cryptosporidium infection [14], a prevalence estimation based on the results of a single diagnostic assay would have been unreliable. In this study for example, the apparent calf prevalence ranged from $13 \%$ to $46 \%$, depending on the technique being used. Previously a Bayesian approach has proven its potential to circumvent this gold standard problem when $3[11,26]$ or 4 tests [5] were used to diagnose infection. The four-test approach was also used in this study and yielded a Cryptosporidium calf prevalence estimate of $17 \%$ (95\% PI: $0.1-0.28$ ), which is comparable to observations by others in calves of the same age category $[16,20,25,32]$. The $95 \%$ PI might even underestimate the prevalence due to intra-herd correlation not being taken into account. When the test results of two additional PCR assays were combined with the results of the four conventional assays and used to develop the six-test Bayesian approach, the prevalence estimate increased to $58 \%$ (95\% PI: $0.5-$ 0.66). The higher prevalence was largely due to the detection by both PCR assays of positive calves older than one month that were not detected by the conventional techniques, which is probably due to the combined effect of the lower oocyst excretion in these calves and the higher sensitivity of PCR compared to the conventional techniques [40]. Similar to previous reports [22, 24, 42], the peak in oocyst excretion in this study was observed in calves between 1 to 4 weeks of age. Although the oocyst excretion decreases in calves older than one month, the prevalence estimated by both PCR assays remained high in calves older than one month, on the contrary to other studies with a decrease in prevalence found in calves in the age category of 4 to 10 weeks $[25,28]$. The results of the present study indicate that calves between the age of 4 to 10 weeks can be an important source of infection for susceptible animals, although there is a possible shift in Cryptosporidium genotypes with age in calves [31].

The evaluation of the conventional techniques in the four-test approach yielded comparable sensitivity estimates for the ME and two out of the three immunological techniques, confirming previous reports that immunological detection methods are not significantly more sensitive than ME $[14,29]$. The specificity of the immunological techniques, however, was higher than the specificity of ME. The speculated higher sensitivity of the PCR assays [19,33] was confirmed by the sixtest approach, with sensitivity estimates for 
the C-PCR and COWP PCR of 0.79 and 0.59 respectively, whereas all other techniques had sensitivity estimates $\leq 0.4$. The difference in test characteristics estimates of the conventional assays determined in the four-test and the six-test approach, was due to the addition of two diagnostic techniques with a high sensitivity into the six-test approach: both PCR assays not only confirmed most samples positive by the four conventional assays, but also detected additional positive samples, resulting in a decrease in sensitivity estimates for the conventional techniques, whereas the specificity estimates for the four conventional assays were comparable in both approaches.

These results also illustrate the pitfalls of test evaluation and prevalence estimation using Bayesian analysis. Similar to diagnostic test evaluation in a frequentist approach, extrapolation of results on test characteristics beyond the limits of the study cannot be made in a Bayesian approach and results should always be considered within the limits of the study. In a Bayesian analysis, the posterior estimates are not rigid parameters but estimated variables resulting from both prior information and the data at hand [5]. Prior information or constraints are essential in the analysis in order to reduce the number of parameters to be estimated. Both in the four-test approach and in the six-test approach, the use of prior information was needed to constrain the parameter space, allowing estimation of all remaining parameters. Expert opinion or published results from previous studies can be used as valuable prior information $[2,36,37]$. In the four-test approach the use of wide range constraints [6] based on previously published estimates of Cryptosporidium prevalence $[16,25,27]$ and test characteristics [14] or on information provided by the manufacturer of the diagnostic assays, was sufficient to reduce the number of parameters to be estimated. In the six- test approach however, the use of more stringent constraints was necessary to reduce the high number of parameters. Although the validity of the prior information used in the six test approach was previously described $[18,33]$ and confirmed by sequencing PCR amplification products of randomly selected samples throughout the study, the posterior estimates of both the prevalence and the test characteristics are not stringent parameters, but should be considered as best-possible estimates based on the observations and the prior information. Since the data at hand in the present study resulted from an epidemiological study including both calves with and without clinical symptoms, and greatly depended on the choice of test for diagnosis, the posterior estimates of the test characteristics must be interpreted taking these limits into account. The rather low sensitivity estimates in the six-test model of the conventional techniques was mostly due to the low excretion of Cryptosporidium oocysts in calves older than one month and to the higher sensitivity of PCR compared to more conventional techniques. It does, however, not imply that these conventional techniques are not reliable for clinical diagnosis, since clinical symptoms are mostly diagnosed in calves younger than one month and correlate with an increased excretion of oocysts. In view of our epidemiological study, however, the results of the six-test approach demonstrate that PCR assays can provide additional information compared to the conventional diagnostic tools.

The results of this study illustrate the benefit of a Bayesian approach in estimating prevalence in a population with unknown disease status and in providing a reliable tool for the evaluation of diagnostic test characteristics, within the limits of the data at hand and the prior information. The need for sensitive diagnostic assays, such as the PCR assays, in epidemiological studies was demonstrated, especially 
for the identification of subclinically infected animals who might be an important source of infection for other animals, since the PCR assays identify those animals with reduced oocyst excretion that the conventional techniques fail to identify.

\section{ACKNOWLEDGEMENTS}

The authors would like to thank Janssen Animal Health for their financial support and $\mathrm{Mr}$ Stijn Casaert for technical assistance.

\section{REFERENCES}

[1] Boelaert M., Aoun K., Liinev J., Goetghebeur E., Van der Stuyft P., The potential of Latent Class Analysis in diagnostic test validation for canine Leishmania infantum infection, Epidemiol. Infect. 123 (1999) 499-506.

[2] Branscum A.J., Gardner I.A., Johnson W.O., Estimation of diagnostic-test sensitivity and specificity through Bayesian modeling, Prev. Vet. Med. 68 (2005) 145-163.

[3] Carreno R.A., Martin D.S., Barta J.R., Cryptosporidium is more closely related to the gregarines than to coccidia as shown by phylogenetic analysis of apicomplexan parasites inferred using small-subunit ribosomal RNA gene sequences, Parasitol. Res. 85 (1999) 899-904.

[4] De Graaf D.C., Vanopdenbosch E., OrtegaMora L.M., Abbassi H., Peeters J.E., A review of the importance of cryptosporidiosis in farm animals, Int. J. Parasitol. 29 (1999) 1269-1287.

[5] Dorny P., Phiri I.K., Vercruysse J., Gabriel S., Willingham III A.L., Brandt J., Victor B., Speybroeck N., Berkvens D., A Bayesian approach for estimating values for prevalence and diagnostic test characteristics of porcine cysticercosis, Int. J. Parasitol. 34 (2004) 569-57.

[6] Dunson D.B., Commentary: practical advantages of Bayesian analysis of epidemiologic data, Am. J. Epidemiol. 153 (2001) 12221226.

[7] Fayer R., Morgan U., Upton S.J., Epidemiology of Cryptosporidium: transmission, detection and identification, Int. J. Parasitol. 30 (2000) 1305-1322.
[8] Garcia L.S., Shimizu R.Y., Evaluation of nine immunoassay kits (enzyme immunoassay and direct fluorescence) for the detection of Giardia lamblia and Cryptosporidium parvum in human faecal specimens, J. Clin. Microbiol. 35 (1997) 1526-1529.

[9] Gelman A., Rubin D.B., Inference from iterative simulation using multiple sequences, Stat. Sci. 7 (1992) 457-511.

[10] Gelman A.E., Carlin J., Stern H., Rubin D., Bayesian Data Analysis, 2nd ed., Chapman and Hall/CRC, Boca Raton, 2004, p. 172.

[11] Geurden T., Claerebout E., Vercruysse J., Berkvens D., Estimation of diagnostic test characteristics and prevalence of Giardia duodenalis in dairy calves in Belgium using a Bayesian approach, Int. J. Parasitol. 34 (2004) 1121-1127.

[12] Heine J., An easy technique for the demonstration of Cryptosporidia in faeces, J. Vet. Med. B 29 (1982) 324-327.

[13] Ignatius R., Eisenblätter M., Regnath T., Mansmann U., Futh U., Hahn H., Wagner J., Efficacy of different methods for detection of low Cryptosporidium parvum oocyst numbers or antigen concentrations in stool specimens, Eur. J. Clin. Microbiol. 16 (1997) 732-736.

[14] Kehl K.S.C., Cicirello H., Havens P.L., Comparison of four different methods for detection of Cryptosporidium species, J. Clin. Microbiol. 33 (1995) 416-418.

[15] Lefay D., Naciri M., Poirier P., Chermette R., Efficacy of halofuginone lactate in the prevention of cryptosporidiosis in suckling calves, Vet. Rec. 148 (2001) 108-112.

[16] Maldonado-Camargo S., Atwill E.R., Saltijeral-Oaxaca J.A., Herrera-Alonso L.C., Prevalence and risk factors for shedding of Cryptosporidium parvum in Holstein Freisian dairy calves in central Mexico, Prev. Vet. Med. 36 (1998) 95-107.

[17] Morgan U.M., O’Brien P.A., Thompson R.C.A., The development of diagnostic PCR primers for Cryptosporidium using RAPDPCR, Mol. Biochem. Parasitol. 77 (1996) 103-108.

[18] Morgan U.M., Constantine C.C., Forbes D.A., Thompson R.C.A., Differentiation between human and animal isolates of Cryptosporidium parvum using rDNA seqencing and direct PCR analysis, J. Parasitol. 83 (1997) 825-830. 
[19] Morgan U.M., Pallant L., Dwyer B.W., Forbes D.A., Rich G., Thompson R.C.A., Comparison of PCR and microscopy for detection of Cryptosporidium parvum in human fecal samples: clinical trial, J. Clin. Microbiol. 36 (1998) 995-998.

[20] Mtambo M.M.A., Sebatwale J.B., Kambarage D.M., Muhairwa A.P., Maeda G.E., Kusiluka L.J.M., Kazwala R.R., Prevalence of Cryptosporidium spp. oocysts in cattle and wildlife in Mororogo region, Tanzania, Prev. Vet. Med. 31 (1997) 185-190.

[21] Newman R.D., Jaeger K.L., Wuhib T., Lima A.M.M., Guerrant R.L., Sears C.L., Evaluation of an antigen capture enzymelinked immunosorbent assay for detection of Cryptosporidium oocysts, J. Clin. Microbiol. 31 (1993) 2080-2084.

[22] Nydam D.V., Wade S.E., Schaaf S.L., Mohammed H.O., Number of Cryptosporidium parvum oocysts or Giardia spp. cysts shed by dairy calves after natural infection, Am. J. Vet. Res. 62 (2001) 1612-1615.

[23] O’Donoghue P.J., Cryptosporidium and cryptosporidiosis in man and mammals, Int. J. Parasitol. 25 (1995) 139-195.

[24] O’Handley R.M., Cockwill C., McAllister T.A., Jelinski M., Morck D.W., Olson M.E., Duration of naturally acquired giardiosis and cryptosporidiosis in dairy calves and their association with diarrhea, J. Vet. Med. Assoc. 214 (1999) 391-396.

[25] Olson M.E., Guselle N.J., O’Handley R.M., Swif M.L., McAllister T.A., Jelinski M.D., Morck D.W., Giardia and Cryptosporidium in dairy calves in British Columbia, Can. Vet. J. 38 (1997) 703-706.

[26] Pouedet M.S.R., Zoli A.P., Nguekam, Vondou L., Assana E., Speybroeck N., Berkvens D., Dorny P., Brandt J., Geerts S., Epidemiological survey of swine cysticercosis in two rural communities of West-Cameroun, Vet. Parasitol. 106 (2002) 45-54.

[27] Quilez J., Sanchez-Acedo C., Clavel A., del Cacho E., Lopez-Bernad F., Comparison of an acid-fast stain and a monoclonal antibody-based immunofluorescence reagent for the detection of Cryptosporidium oocysts in faecal specimens from cattle and pigs, Vet. Parasitol. 67 (1996) 75-81.

[28] Quilez J., Sanchez-Acedo C., del Cacho E., Clavel A., Causapé A.C., Prevalence of
Cryptosporidium and Giardia infections in cattle in Aragon (Northeastern Spain), Vet. Parasitol. 66 (1996) 139-146.

[29] Rodriguez-Hernandez J., Canutblasco A., Ledesmagarcia M., Martinsanchez A.M., Cryptosporidium oocysts in water for human consumption. Comparison of staining methods, Eur. J. Epidemiol. 10 (1994) 215-218.

[30] Rosenblatt J.E., Sloan L.M., Evaluation of an enzyme-linked immunosorbent assay for detection of Cryptosporidium spp. in stool specimens, J. Clin. Microbiol. 31 (1993) 1468-1471.

[31] Santin M., Trout J.M., Xiao L., Zhou L., Greiner E., Fayer R., Prevalence and agerelated variation of Cryptosporidium species and genotypes in dairy calves, Vet. Parasitol. 122 (2004) 103-117.

[32] Sischo W.M., Atwill E.R., Lanyon L.E., George J., Cryptosporidia on dairy farms and the role these farms may have in contaminating surface water supplies in the northeastern United States, Prev. Vet. Med. 43 (2000) 253-267.

[33] Spano F., Putignani L., McLaughlin J., Casemore D.P., Crisanti A., PCR-RFLP analysis of the Cryptosporidium oocyst wall protein (COWP) gene discriminates between $C$. wrairi and $C$. parvum, and between $C$. parvum isolates of human and animal origin, FEMS Microbiol. Lett. 150 (1997) 209-217.

[34] Spiegelhalter D.J., Best N.G., Carlin B.P., der Linde A., Bayesian measures of model complexity and fit (with discussion), J. R. Stat. Soc. B 64 (2002) 583-640.

[35] Spiegelhalter D.J., Thomas A., Best N.G., Lunn D., WinBUGS Version 1.4 User Manual, vol. 4, MRC Biostatistics Unit, Cambridge, 2003.

[36] Suess E.A., Gardner I.A., Johnson W.O., Hierarchical Bayesian model for prevalence inferences and determination of a country's status for an animal pathogen, Prev. Vet. Med. 55 (2002) 155-171.

[37] Toft N., Jorgensen E., Hojsgaard S., Diagnosing diagnostic tests: evaluating the assumptions underlying the estimation of sensitivity and specificity in the absence of a gold standard, Prev. Vet. Med. 68 (2005) 19-33.

[38] Wade S.E., Mohammed H.O., Schaaf S.L., Prevalence of Giardia spp., Cryptosporidium parvum and Cryptosporidium muris (C. andersoni) in 109 dairy herds in five counties 
of southeastern New York, Vet. Parasitol. 93 (2000) 1-11.

[39] Weber R., Bryan R.T., Bishop H.S., Wahlquist S.P., Sullivan J.J., Juranek D.D., Treshold of detection of Cryptosporidium oocysts in human stool specimens: evidence for low sensitivity of current diagnostic methods, J. Clin. Microbiol. 29 (1991) 1323-1327.

[40] Webster K.A., Smith H.V., Giles M., Dawson L., Robertson L.J., Detection of Cryptosporidium parvum oocysts in faeces: comparison of conventional coproscopical methods and the polymerase chain reaction, Vet. Parasitol. 61 (1996) 5-13.

[41] Xiao L., Herd R.P., Quantitation of Giardia cysts and Cryptosporidium oocysts in fecal samples by direct Immunofluorescence assay, J. Clin. Microbiol. 31 (1993) 2944 2946.

[42] Xiao L., Herd R.P., Infection patterns of Cryptosporidium and Giardia in calves, Vet. Parasitol. 55 (1994) 257-262.

To access this journal online: www.edpsciences.org 